\title{
Current management of enterocutaneous fistulas
}

\author{
R. Latifi $\cdot$ F. Turégano
}

Received: 2 February 2011 / Accepted: 4 March 2011 / Published online: 29 March 2011

(C) Springer-Verlag 2011

The editors of the European Journal of Trauma and Emergency Surgery asked us to put together a focus issue on the current management of gastroenterocutaneous fistulas. As surgeons who deal almost daily with this problem, we were delighted to take this on, but the task before us was not simple. The problem is enormous for the individual patient and treating surgeon, which requires real expertise, complex surgical intervention, and a team approach. With this in mind, we set to create a series of articles that will meet one aim: review the current principles and practices of the management of this serious clinical issue and, perhaps most importantly, articulate whether there is anything new in the management of fistulas both from the surgical standpoint, as well as from the medical and physiology standpoint. For this, we asked many of our colleagues, all practicing surgeons, from different parts of the world, to help us address these questions. So is there anything new, indeed? We will let the readers decide on that.

Since the first documented enterocutaneous fistula (ECF) that dates back to 1822 when a man named Alexis St. Martin suffered a musket shot to the chest and abdomen, the science and surgical practice of fistulas has changed significantly.

R. Latifi $(\bowtie)$

Department of Surgery, University of Arizona, Tucson, Arizona

e-mail: rlatifi@email.arizona.edu

R. Latifi

Hamad Medical Corporation, Department of Trauma and Critical

Care, Doha, Qatar

F. Turégano

General Surgery II Service and Emergency Department (Surgical Section), University General Hospital Gregorio Marañón,

Madrid, Spain
What is well known is the fact that few ECF develop spontaneously, occurring typically in the face of inflammatory bowel disease, malignancy, radiation, and diverticulitis. Most commonly, however, ECF are iatrogenic and postoperative. Fistulas, be they enterocutaneous, enteroatmospheric (EAF), enteroenteric, or entero-some other organ, are easy to recognize, but it is much more difficult to treat them. Those of us who treat patients with this malady know first hand that the more you operate, the more potential complications they develop, yet, for the most part, unless fistulas close spontaneously, they will need complex surgical intervention, and, often, multiple operations. Treating well-recognized characteristic sequelae of ECF such as sepsis, malnutrition, and fluid and electrolyte disturbances before definitive surgical intervention remains the cornerstone of the management of fistulas. These largely remain relevant today and are echoed throughout all of the papers of this focus.

What is really new in the treatment of these complex patients is our ability to provide complete nutrition support using total parenteral nutrition (TPN) for as long as it takes, use complex surgical techniques to provide skin coverage, abdominal wall reconstruction using both tissue transfer techniques and biological mesh, and use wound vacuumassisted closure (VAC) in both patient and outpatient settings. These three treatment modalities are the most important elements that have become part of surgeons' armamentarium in caring for patients with fistulas. All of these principles have been described clearly in this focus issue. One other important element is the fact that, radiographically, we can diagnose and identify the anatomy of the disease better than ever, and we can treat many of the infectious complications less invasively than the major surgeries which these patients often require. Obviously, we have powerful antibiotics and anti-fungal therapy to help treat life-threatening infections as well. 
To these principles and 'new' approaches from the early days, we should add our understanding of the importance of the timing of surgical intervention. Thus, surgeons have developed a logical stepwise order to the treatment of ECF, and this is as follows: prevent and/or control of sepsis, manage fluids and electrolytes imbalance, protect the skin, define anatomy, and plan surgical intervention. Most important though, we have a multidisciplinary team that takes care of these patients. While the surgeon should be the team leader, and should direct the treatment, there are many others with as much importance, if not more, than surgeons, who are an integral part of the treatment of these patients. In reality, there is much overlap, with multiple steps occurring simultaneously. The same principles are true for EAF, with the addition of special considerations to wound care and abdominal wall closure during definitive surgical intervention.
Despite all of the advances made in surgery, the mortality from fistulas is still high, morbidity is serious, financial burden on medical systems enormous, and loss of productivity from fistulas is measurable. To this end, hopefully, this focus on fistulas has shed some light on recognizing the risk factors for the development of fistulas, techniques for identifying the anatomy of the disease, and, most importantly, treating these complex patients using different surgical and medical techniques. One factor should be reiterated as well. These patients should be treated by surgeons who have developed experience and expertise and have an interest, both surgical and biological, in treating these complex patients. Otherwise, the consequences are serious and the complications enormous. 